\author{
Hans Peter Bruggisser
}

Viele Krankheiten oder diagnostische Beschreibungen von Krankheitsphänomenen scheinen an einen jeweiligen Zeitgeist oder an Modeströmungen gebunden zu sein, insbesondere die psychiatrischen Diagnosen. Depression und Burnout gehören $\mathrm{zu}$ den (Schlag-)Wörtern unseres Jahrhunderts. Jedermann glaubt, sich mit diesen Begriffen bestens auszukennen und hat dann im konkreten Fall gleichwohl Probleme, diese Phänomene konzise $\mathrm{zu}$ formulieren. Vielleicht auch deshalb, weil allein das BurnoutSyndrom («ausgebrannt sein») ca. 130 Symptome umfasst, welche sich mit der Depressionskrankheit grossenteils decken.

Diese modernen Krankheitsbegriffe können jedoch nicht negiert oder verschwiegen werden. Allein schon durch die Tatsache, dass nach einigen Statistiken bis $\mathrm{zu}$ 16\% der

\title{
Depression und Burnout
}

Mit diesen beiden Krankheitsbegriffen soll dargestellt werden, dass sie Endergebnis oder Folgen unseres modernen Lebenswandels sein können. Zudem stehen sie miteinander in engem Zusammenhang, und beide führen zu einer Art Erschöpfung. Stress und das Durchbrechen von Regelkreisen führen zur Entwicklung dieser Phänomene. Eine Anhäufung von Lebensereignissen oder Mikrotraumata führen zu einer psychosomatischen Reaktion. Prophylaxe und Therapiekonzepte werden aufgezeigt.

schweizerischen Arbeitsbevölkerung unter einem Burnout leiden sollen und dass diese Krankheit gegenwärtig eher noch im Zunehmen begriffen ist.

\section{Versuch einer ganzheitlichen Diagnostik}

Wenn wir versuchen, uns auf diese Begriffe näher einzulassen, kann eine Übersicht von Burisch weiterhelfen. In Tabelle 1 wird in Spalte 1 einerseits der Zusammenhang zwischen Depression und Burnout erkennbar und als ein Entwicklungsprozess dargestellt. Beginnend bei chronischem Stress führt er hin zu Burnout und von einer depressiven Symptomatik hin zur klinischen Depression. Andererseits kann man sich in dieser kurzen Übersicht schnell über die verschiedenen Phasen eines Burnouts und die dazugehörende Symptomatik orientieren.

Wie in Tabelle 1 erwähnt, ist neben dem Burnout und der Depression der weitere Begriff «Stress» von Bedeutung, zumal er als Ausgangspunkt und Ursache unserer Thematik betrachtet werden kann. Eine der vielen existierenden Definitionen beschreibt den Begriff Stress als Gefühl, welches immer dann entsteht, wenn Menschen den Eindruck haben, es wachse ihnen etwas über den Kopf, eine Situation könne nicht mehr kontrolliert werden.

Als «Menschen in der Lebensmitte» werden neuerdings jene Alterskategorien bezeichnet, welche nach einer umfassenden Ausbildung in die Arbeitswelt eintreten, sich dort um eine Berufskarriere kümmern und gleichzeitig die Gründung einer Familie nicht vergessen sollten. Die Kinder

Tab. 1. Die Phasen des Burnout-Syndroms nach Burisch

\begin{tabular}{|c|c|c|}
\hline Entwicklung & Phase & Symptome \\
\hline Chronischer Stress & 1. Erste Warnzeichen & $\begin{array}{l}\text { Gesteigerter Einsatz für Ziele. Überstunden nehmen zu mit Erschöpfung und } \\
\text { vegetativen Symptomen }\end{array}$ \\
\hline Burnout & 2. Reduziertes Engagement & $\begin{array}{l}\text { Reduzierte soziale Interaktion, negative Einstellung zur Arbeit, Konzentration } \\
\text { auf eigenen Nutzen }\end{array}$ \\
\hline \multirow[t]{4}{*}{ Depressive Symptomatik } & 3. Emotionale Reaktionen & $\begin{array}{l}\text { Insuffizienzgefühle, Pessimismus, Leere, Hoffnungslosigkeit, Energiemangel, } \\
\text { Gefühl von Hilflosigkeit, Schuldzuschreibung an andere }\end{array}$ \\
\hline & 4. Abnahme ... & $\begin{array}{l}\text {... von kognitiven Fähigkeiten, Motivation, Kreativität und } \\
\text { Differenzierungsfähigkeit }\end{array}$ \\
\hline & 5. Abflachen ... & $\ldots$ des emotionalen und sozialen Lebens und kognitiver Interessen \\
\hline & 6. Psychosomatische Reaktionen & $\begin{array}{l}\text { Spannung, Schmerzen, Insomnie, Inappetenz, in Freizeit ist keine Erholung } \\
\text { mehr möglich, Veränderung der Essgewohnheiten, Substanzgebrauch }\end{array}$ \\
\hline Klinische Depression & 7. Depression und Verzweiflung & $\begin{array}{l}\text { Gefühl von Sinnlosigkeit, negative Lebenseinstellung, existenzielle } \\
\text { Verzweiflung, Suizidgedanken oder Absichten }\end{array}$ \\
\hline
\end{tabular}

\section{KARGER}

Fax +497614520714 Information@Karger.d www.karger.com (c) 2010 S. Karger GmbH, Freiburg

Accessible online at: www.karger.com/szg
Dr. med. Hans Peter Bruggisser

Leitung Psychosomatik, Psychotherapie und Psychiatrie

Aeskulap-Klinik

Gersauerstrasse 8, 6440 Brunnen, Schweiz

Tel. +41 825-4747, Fax -4965

HansPeter.Bruggisser@aeskulap.com 
brauchen nicht nur ein Zuhause, sondern fordern von Eltern ein immer grösseres Engagement für deren künftige Autonomie. Mit der zunehmenden Alterung der Menschheit müssen sich die Menschen in der Mitte zusätzlich um ihre eigenen Eltern kümmern, wenn diese ihre Autonomie wieder verlieren. Solche Zeitphänomene und Engagements können einem Individuum, männlich und weiblich, tatsächlich über den Kopf wachsen. Sie fördern Stress und Erschöpfungssituationen, welche in einem Burnout oder in einer Depression enden können. Solche Menschen haben ihre innere Balance verloren.

Prof. Nossrat Peseschkian hat ein Balance-Modell entwickelt, das diese Tatsachen ganzheitlich zu umfassen versucht. Um in einem Gleichgewicht $\mathrm{zu}$ bleiben, sollte im Idealfall eine gleichmässige Verteilung in vier grosse Lebensbereiche stattfinden können, welche im Alltag immer wieder überprüft und ausgeglichen werden müssten. Es geht um die Bereiche:

1. Körper/Sinne (Wie nehme ich meinen Körper wahr und wie gehe ich mit ihm um?)

2. Bereich Arbeit/Leistung (Wie viel Arbeit leiste ich und mit welcher Motivation?)

3. Bereich Kontakt/Familie (Habe ich ausreichend erfüllende Beziehungen?)

4. Bereich Zukunft/Phantasie (Verfüge ich über Projekte, ergibt mein Leben einen Sinn?).

In Abbildung 1 sind die vier Bereiche ersichtlich. Die gestrichelt eingezeichnete Raute zeigt das Bild einer Idealverteilung der Energien und das rechtschenklige Dreieck zeigt das Bild eines typischen Burnout-Patienten. Für den Bereich Arbeit und Leistung verbrauchen diese Menschen zu viel Energie, welche in der Folge in den übrigen Bereichen fehlt.

Erschöpfungszustände und Depressionen sind nur selten einem einzelnen, bestimmten Lebensereignis oder Trauma zuzuordnen. Die Ur- sachen sind meist multifaktoriell, und es muss nach sog. «Mikrotraumen» gesucht werden, wie sie eben im Verlaufe eines Lebens manchmal gehäuft auftreten können. In Abbildung 2 sind für die einzelnen Bereiche einige solcher typischen Ereignisse aufgezählt, welche rückblickend auf die letzten
10 Jahre schnell einmal die Anzahl von einem halben Dutzend erreichen können und Ausmasse annehmen, die für ein Individuum ein Mikrotrauma bedeuten können.

Die Veranlagung eines Individuums, die jeweilige Lebensphase mit ihren spezifischen Stressoren und die

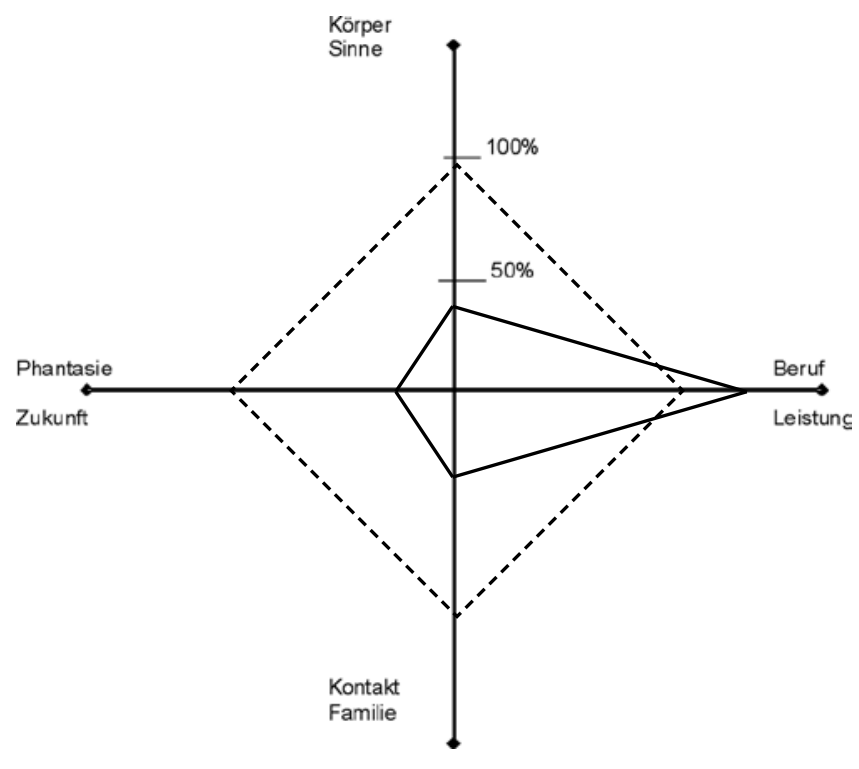

Abb. 1. Das BalanceModell nach Peseschkian.

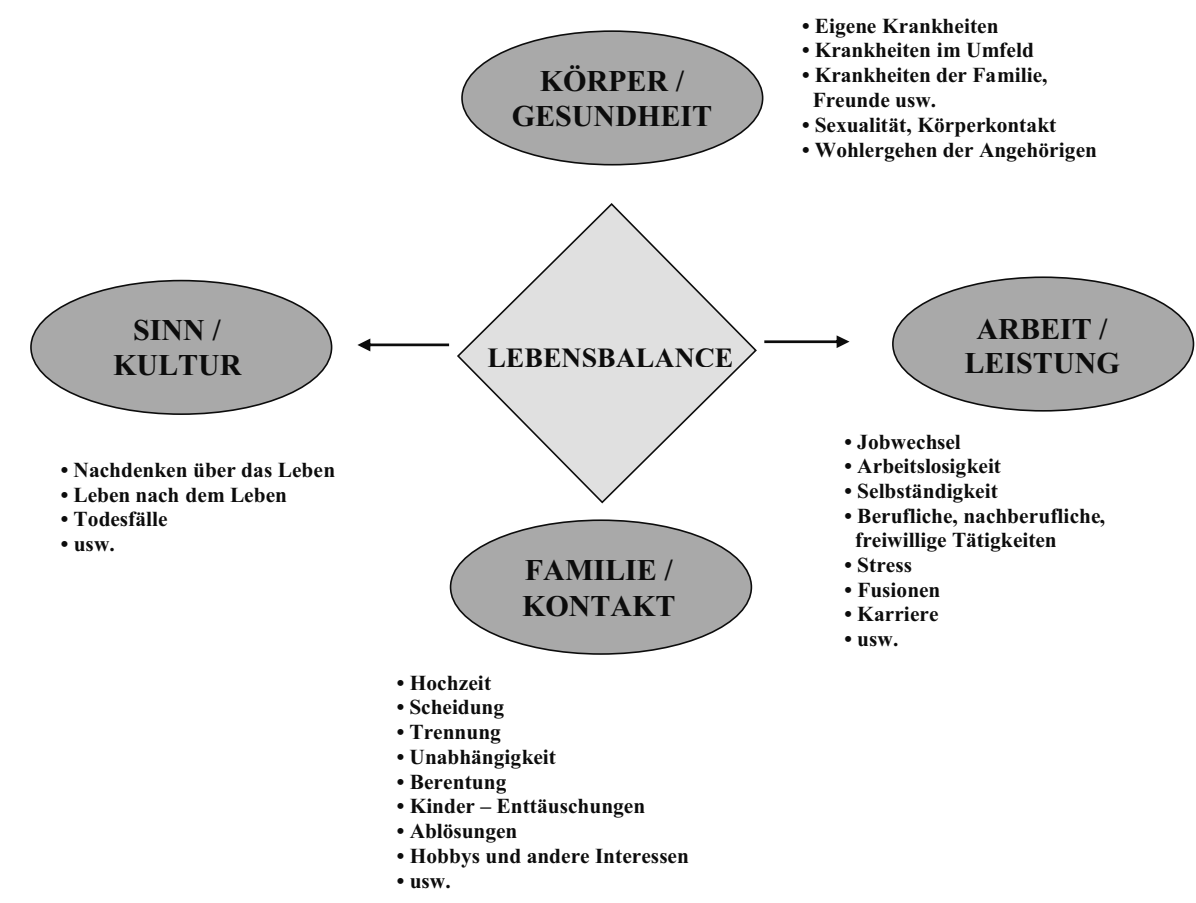

Abb. 2. Life Events nach Peseschkian. 
Anzahl solcher Mikrotraumen führen bei inadäquater Verarbeitung unweigerlich zu einer Einschränkung der Lebensqualität oder eben zu einer sog. «psychosomatischen Reaktion», welche das Auftreten von Erschöpfungszuständen, sei es eine Depression oder ein Burnout-Syndrom, erklären. Die Abbildung zeigt, dass das Auftreten von Lebensereignissen mit den uns gegebenen Konzepten und der bereits gewonnenen Lebenserfahrung aufgefangen werden können und sich psychisch/seelisch als kürzer oder länger dauernde «innere Unruhe» bemerkbar machen und wieder abklingen.

Wenn aber die Anzahl dieser «life events» ein bestimmtes Mass überschreitet, führt dies zu Stress, welcher sich störend auf den Hirnstoffwechsel auswirkt. Letzterer führt $\mathrm{zu}$ einer Auslenkung des NeurotransmitterSystems und im Bereich des vegetativen Nervensystems zu sog. funktionellen Beschwerden. Die Aufzählung dieser vegetativ bedingten Beschwerden führt bei vielen Betroffenen in der Regel zu einem «Aha-Erlebnis». Bei dieser Gelegenheit soll darauf hingewiesen werden, dass das Thema Depression in diesem Beitrag einzig unter dem Aspekt der «Erschöpfung» oder des «Energiemangels» betrachtet wird.

Bei einem Burnout-Syndrom kommen noch arbeitsspezifische Faktoren dazu, wie z.B.:

- hohe Arbeitsbelastung,

- Zeit- und Verantwortungsdruck,

- Streben nach Perfektion und sozialer Anerkennung,

- wachsende Komplexität und Unüberschaubarkeit von Arbeitsabläufen und Reizüberflutung,

- unpersönliches, bedrückendes oder intrigenbelastetes Arbeitsklima.

Soweit der Versuch, die Phänomene Burnout und Depression einerseits unter dem Aspekt der Erschöpfung sowie deren Ursachen und Hintergründe zu betrachten. In diesem Sinne wird das Burnout von der Depres- sion lediglich abgegrenzt als eine spezifische Erschöpfung mit arbeits- und berufsspezifischen Stressfaktoren.

Andererseits soll das Ganzheitliche miteinbezogen werden, vorerst einmal im Sinne, dass Körper und Geist/Seele zwar wissenschaftlich getrennt werden, in der Realität jedoch in einem feingliedrigen Regelwerk miteinander in Verbindung stehen. Dies soll bei der Darstellung in Abbildung 3 nach Peseschkian wieder verdeutlicht werden. Das Ganzheitliche spielt in der Folge auch eine wichtige Rolle in therapeutischer Hinsicht.

\section{Ganzheitliche therapeutische Gesichtspunkte}

Die vier Grundpfeiler einer ganzheitlichen Therapie von Erschöpfungszuständen umfassen: Stressbewältigung, Revitalisierung, Bewegung und Ernährung.

\section{Stressbewältigung}

Mit individueller Psychotherapie und Psychoedukation (individuell oder eventuell in Kleingruppen). In der Psychotherapie geht es um die Aufarbeitung der erwähnten Mikrotraumata, nach Methode des jeweiligen
Therapeuten oder mit der Therapiemethode nach Peseschkian (die fünf Therapieschritte).

Beim Burnout kommen spezifische, arbeitsbezogene Faktoren dazu, wie z.B.:

- Zieldefinitionen, welche realistisch angepasst werden müssen.

- Selbstdefinition, was man als Mensch vom Leben und seiner Arbeit erwartet.

- unterscheiden, was die eigene Arbeit befruchtet und was sie verschwendet.

- Grenzen setzen, authentische Offenheit gegenüber den eigenen Gefühlen.

In der Psychoedukation muss ein betroffener Patient wieder erlernen, seinen Körper wahrzunehmen, auf die zahlreichen Warnsignale zu achten und diese richtig zu interpretieren. Ebenso wichtig ist das Kennenlernen von verschiedenen Entspannungstechniken und deren Auswirkungen auf Stressbelastung. Dabei gilt es zusätzlich herauszufinden, welche Methode man individuell bevorzugt und später eventuell selbst anwenden kann.

Ob Menschen ein Ereignis als unkontrollierbar, d.h. als Stress erleben, bestimmen sie durch die ganz persönliche Bewertung des Ereignisses. Men-

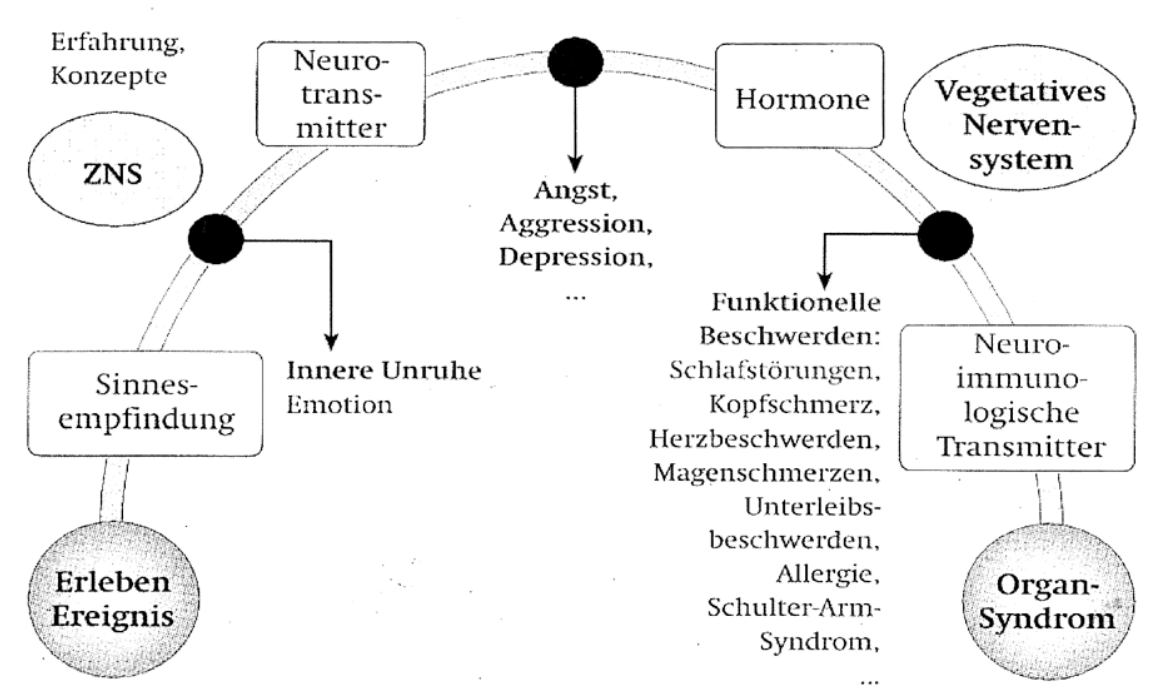

Abb. 3. Die psychosomatische Reaktion nach Peseschkian. 
schen, deren Stressreaktion durch innere Faktoren hervorgerufen wird, haben sich viele Denkgewohnheiten zugelegt, die ihnen auch dann Stress erzeugen, wo es gar nicht notwendig wäre, d.h., sie sehen Gefahren, wo keine Gefahren sind, überbewerten diese und unterschätzen die eigenen Fähigkeiten, damit zurande zu kommen. Stressbearbeitung ist daher fast immer das Verändern und Erlernen neuer Denkgewohnheiten und neuer Denkmuster.

Wie in Abbildung 3 aufgezeigt, führt eine andauernde Stressbelastung zu einer Neurotransmitter-Auslenkung, welche ab einem bestimmten Ausmass und einer spezifischen Symptomatik medikamentös behandelt werden sollte. In der Ganzheitsmedizin hat sich in diesem Sinne das Johanniskraut (Hypericum) als Mittel der Wahl für depressive Erschöpfungszustände eingebürgert, zumal dessen Wirksamkeit gemäss neuer Metaanalysen (Cochrane) mehrfach nachgewiesen wurde. In einigen Fällen sind jedoch klassische Antidepressiva noch immer indiziert. Für eine medikamentöse Stressreduktion sind weitere gut wirksame Phytotherapeutika als Monosubstanzen oder Kombinationen auf dem Markt. Für Erschöpfungszustände oder chronische Stressphänomene hat sich auch die Anwendung mit Magnetfeldtherapie bewährt.

\section{Revitalisierung}

Depression und Burnout blockieren nicht nur psychische, sondern auch physische Regelkreise. Ersatz von Vitaminen, Spurenelemente und der Einsatz von Ozoninfusionen lösen solche Blockaden und verbessern das Energiepotenzial innerhalb kurzer Zeit wesentlich. Die zusätzliche An- wendung von Colon-Hydro-Therapie und die phytotherapeutische Anregung des Leberkreislaufs tragen zusätzlich zur Revitalisierung bei, indem die Immunreaktion des Körpers gestärkt wird. Interessant in diesem $\mathrm{Zu}$ sammenhang ist der Hinweis, dass die Bildung der Immunabwehr und auch der Neurotransmitter zum grossen Teil in einem gut funktionierenden Darm stattfinden.

Ein wichtiger Anreiz zur Revitalisierung kann auch die Anwendung von Wärme im Sinne einer aktiven oder passiven Hyperthermie bedeuten. Das sind altbekannte Methoden, welche heute aufgrund neuer Techniken wieder mit Erfolg angewendet werden. Eine vorangehende 24-hKörperinnentemperaturmessung gibt die notwendigen Hinweise für Ausschlusskriterien.

\section{Bewegung}

Das alte Sprichwort des römischen Dichters Juvenal vom «gesunden Geiste in einem gesunden Körper» bezieht sich auch auf eine ausgeglichene Körperbetätigung. Wichtig dabei ist, den Schweregrad der körperlichen Erschöpfung zu erfassen, um aufgrund dieser Werte ein individuell «massgeschneidertes», körperliches Aufbauprogramm aufzustellen. Nebst vielen Laborhinweisen (Eisenmangel, Schilddrüsenunterfunktion und chronische Entzündungen!) ist die Messung der Herzratenvariabilität und eine Leistungsanalyse mit Laktatmessung von grossem Nutzen. Sehr oft besteht die Notwendigkeit, dass Patienten wegen eines enorm erhöhten Parasympathikotonus vorerst einmal in Sachen Entspannung instruiert werden, bevor man sie mit einem ausgeklügelten Trainingsprogramm «auf die Piste schickt».

\section{Ernährung}

Auch auf diesem Gebiet eröffnet sich ein weites Feld sich immer wieder verändernder diätetischer Ratschläge und Wechselspiele. Am besten verschwört man sich auf keines im absoluten Sinne. Auch in Sachen Ernährung sei wieder einmal darauf hingewiesen, dass damit mehr gemeint ist, als alleine dem Körper die notwendigen Nährstoffe zuzuführen. Die Ernährung, das Essen, ist auch ein soziales Element. Es wird bekömmlicher, wenn man es in Gesellschaft zu sich nimmt. Hat man dazu keine Gelegenheit, sollte man zumindest darauf achten, dass der Akt des Essens zumindest als bewusster Prozess wahrgenommen wird. Bewusst und meditativ essen, wie eine Schreitmeditation. Das Essen vielfältig gestalten mit der ausgewogenen Verteilung der Bestandteile nach neuestem Wissen. Dazu gehört in der Regel mehr Gemüse und "Grünfutter», als man es gewohnt ist. Damit ist noch ein anderer wichtiger Gesichtspunkt angesprochen: Das Essen sollte mit reichlich Farben gestaltet werden: Gemüse und Zutaten so auswählen, dass ein Teller mit Essen gefüllt einer Palette froher Farben entspricht.

\footnotetext{
Literatur

Burisch M: Das Burnout-Syndrom, ed 3. Heidelberg, Springer, 2005.

Perrig-Chiello P: In der Lebensmitte. Die Entdeckung des mittleren Lebensalters. Zürich, NZZ Libro, 2008.

Peseschkian N: Psychosomatik und positive Psychotherapie. Frankfurt/M., Fischer, 1993.

Schulze B, Rössler W: Burnout Syndrom. Diagnose und Therapie in der klinischen Praxis. Neurologie \& Psychiatrie 2006;4:23-25.

Shirom A, et al: Burnout, mental and physical health: a review of the evidence and a proposed explanatory model. Int Rev Ind Organ Psychol 2005;20:269-309.
} 